\title{
Is mental rotation controlled or automatic?
}

\author{
MICHAEL C. CORBALLIS \\ University of Auckland, Auckland, New Zealand
}

\begin{abstract}
A task requiring the mental rotation of letters was performed either alone or during the retention interval of two short-term memory tasks. The retention of eight digits or random configurations of eight dots slowed overall reaction time on the mental-rotation task, but did not significantly alter the estimated rate of mental rotation. Conversely, performance on the memory tasks did not vary with the angle of mental rotation. These results support the view that attentional control is required to set up the mental structures required in mental rotation, but that the actual execution of the mental-rotation component can be relegated to subordinate systems that do not compete for attentional resources.
\end{abstract}

In a series of experiments, Schneider and Shiffrin (1977; Shiffrin \& Schneider, 1977) documented a case for a fundamental distinction between controlled and automatic processes. Controlled processes are serial and load-dependent and they require active attention, whereas automatic processes require no active control or attention and may be run concurrently with other processes. Although Schneider and Shiffrin's experiments were mainly concerned with the search for memorized items among sequentially presented visual displays, the distinction was held to apply to a broad spectrum of cognitive processes. It has been a matter of some controversy, however, especially in relation to the item-recognition task devised by Sternberg $(1966,1969)$. This task has been dubbed a "paradigm case" for the dichotomy between controlled and automatic processing (Ryan, 1983).

The item-recognition task requires the subject to decide whether or not a presented item is among some prememorized set of items. In the absence of prolonged practice with the same set of items or with different sets characterized by response consistency (Kristofferson, 1977), reaction time (RT) to make such decisions is a linear function of the number of items in the set, at least for sets within the span of immediate memory (cf. Corballis, Katz, \& Schwartz, 1980). Sternberg concluded from this that the subject's decision was accomplished by means of a serial exhaustive scan through the set.

Schneider and Shiffrin (1977; Shiffrin \& Schneider, 1977) regarded this scan as controlled rather than automatic. One difficulty with this is that subjects are typically unaware that they are scanning in this manner, which raises doubts as to whether it can be regarded as truly under their control (Ryan, 1983). Schneider and Shiffrin seem to recognize this problem when they refer to the scan as a veiled rather than an accessible controlled process, but as Ryan (1983) pointed out, this is a label rather than a solution. There is some doubt, moreover, as to whether a serial scan is involved at all in item recognition. Other

Please send reprint requests to: M. C. Corballis, Department of Psychology, University of Auckland, Private Bag, Auckland, New Zealand. models, based, for example, on parallel search (Ratcliff, 1978) or on direct access to the appropriate memory representation (Corballis, Kirby, \& Miller, 1972), can be fitted adequately to the RT data and seem more consistent with automatic rather than with controlled processing.

Logan (1978) examined the influence of a concurrent attention-demanding activity on item recognition. Subjects were required to perform the item-recognition task while holding seven digits in short-term memory for subsequent ordered recall, an activity known to interfere with other choice-RT tasks (e.g., Shulman \& Greenberg, 1971; Shulman, Greenberg, \& Martin, 1971). This activity did tend to increase overall RT for item recognition, and also increased the slope of the function relating RT to memoryset size, suggesting that memory scanning does compete for attentional resources. When subjects practiced with the same memory sets, however, the competing memory task no longer had any effect on the slope, which nevertheless remained significantly above zero. Hence, memory scanning remained apparently serial, yet did not compete for attentional resources, again raising the question of whether it was a controlled or an automatic process. Logan (1978) also found that holding seven digits in shortterm memory had no effect on the other component stages underlying item recognition-namely, the comparison, decision, and response-initiation stages (Sternberg, 1969). Logan argued that attention was involved primarily in the setting up, or "harnessing," of the component processes in item recognition rather than in the actual execution of these processes.

In the present study, the distinction between controlled and automatic processing was examined in relation to the mental-rotation task pioneered by Cooper and Shepard (1973). In the version of the task used here, subjects are required to decide between normal and backward versions of letters presented in different angular orientations. RT to make such decisions is typically a sharply increasing function of the angular departure of the letters from the normal upright, leading Cooper and Shepard to infer that the subjects "mentally rotate" some internal representation of the letters to the upright before making the deci- 
sion. This process is conceptualized as serial, though continuous.

Mental rotation provides an interesting contrast to memory scanning. Whereas subjects are typically unaware of the actual process of memory scanning, most subjects do report introspectively that they carry out mental rotations in deciding whether disoriented characters are normal or backward. Moreover, unlike memory scanning, mental rotation does seem to be under the subject's control, at least in the sense that it can be initiated or stopped at will, or according to instructions. Cooper and Shepard (1973) were even able to instruct their subjects to mentally rotate a letter in synchrony with verbal and visual prompts indicating the required orientation at successive intervals of time, and to verify experimentally that the subjects were actually doing this. On these grounds. one might expect the actual process of mental rotation to be controlled rather than automatic, and thus to be slowed down if it must compete for attentional resources.

Our procedure, like that used by Logan (1978) to assess the attentional demands of item recognition, was to have subjects carry out a task involving the mental rotation of letters while retaining information in short-term memory. There were two competing tasks, one requiring retention of eight digits and the other requiring retention of a pattern of eight dots in a square; there was also a control condition in which there was no competing activity. The spatial retention task was included along with the verbal one in order to test whether mental rotation might exhibit what Fodor (1983) has termed "modularity." Insofar as the mental-rotation component of the Cooper-Shepard task is spatial, it might compete more for resources in the spatial domain than in the verbal domain. If so, then we might expect it to be affected more by the retention of patterns than by the retention of digits, although the retention of digits might have the greater effect on other components of the task, such as the actual identification of the letters.

There is one caveat to be noted, however. Klapp, Marshburn, and Lester (1983) have argued that span memory does not compete for central resources with the working memory of information processing. If this is so, then the attentional demands of a task cannot be assessed by examining the influence of short-term retention on performance of that task. However, Klapp et al. do provide evidence that rehearsal of information in short-term memory does compete with resources for information processing, even if retention per se does not, and that interference is maximal early in the retention interval when rehearsal activity is at a peak. In the present study, the mental-rotation task was presented as soon as practicable after presentation of the material to be held in short-term memory, in an attempt to maximize the potential competition.

\section{METHOD}

\section{Subjects}

The subjects were 2 men and 13 women drawn from a third-year undergraduate course in cognitive psychology. The disproportion- ate number of women reflects the sex ratio in the course as a whole. Later inspection of the results indicated that the performance of the 2 men did not differ in any obvious ways from that of the women. The subjects' ages ranged from 19 to 36 years.

\section{Stimuli and Apparatus}

The stimuli for the mental-rotation task were the uppercase letters F, L, P, and R, generated by an Apple II+ computer and displayed on a Kaga screen. Each letter was $4 \mathrm{~cm}$ high and $2.4 \mathrm{~cm}$ wide. To ensure rapid projection, they were constructed of straight lines, so that the normally rounded portions of the $\mathrm{P}$ and $\mathrm{R}$ were in fact square. They were displayed in six different angular orientations: $0^{\circ}, 60^{\circ}, 120^{\circ}, 180^{\circ}, 240^{\circ}$, and $300^{\circ}$ clockwise from the normal upright. They were presented centrally on the screen and viewed from a distance of about $1 \mathrm{~m}$.

The experiment was controlled by the computer but monitored by the experimenter. The subject sat facing the screen with his or her preferred hand resting on the keys representing the letters $B$ and $\mathrm{N}$ on the console. These letters are adjacent on the bottom row of symbols, with the B to the left of the N. The B was used to signal backward letters and the $\mathrm{N}$ to signal forward letters. Prior to each trial, a message appeared on the screen instructing the subject to press either key when ready. As soon as a button was pressed, the message disappeared and the trial was under way.

Under the control condition, a brief warning tone of about 100 msec duration sounded, and then a fixation cross appeared on the screen for $500 \mathrm{msec}$. It was followed by the stimulus letter, which remained on the screen until the subject signaled whether it was normal or backward. Immediately following the response, the initial message reappeared for the next trial, except that after the last trial, a message appeared telling the subject that the ordeal was over.

Under the digits condition, the initiation response was followed by the presentation of eight digits, randomly selected without replacement from the digits 1 through 9 . They were presented one at a time, from left to right, in the lower left portion of the screen. They appeared at a rate of 2 per second, and each remained on the screen until all eight had been presented. Total presentation time was thus $4 \mathrm{sec}$. Then the digit display disappeared, the warning beep sounded, the fixation cross appeared for $500 \mathrm{msec}$, followed by the stimulus letter, as in the control condition. After the subject responded to the letter, an instruction appeared on the screen telling the subject to type in the digits, using the computer keyboard. Subjects were to type each digit in its correct position, using the zero if they did not remember a particular digit in a particular position. Corrections were permitted using normal keyboard controls, but once the subject was satisfied with the sequence typed in, he or she pressed the return button, and the message to initiate the next trial then appeared on the screen.

Under the dots condition, initiation of a trial was followed immediately by presentation of a square, measuring $4 \times 4 \mathrm{~cm}$ and containing eight dots. There were 25 possible locations for these dots, defined by the intersections of five imaginary vertical with five imaginary horizontal lines which, if placed over the square, would subdivide it into 36 equal squares. The eight dots were selected randomly on each trial, without replacement, from the 25 locations. This display appeared centrally for $2 \mathrm{sec}$, followed by the warning beep, fixation cross, and then the letter as in the control condition. After the subject had responded to the letter, a second display of dots appeared. This either was identical to the first or, with equal probability, was altered by the random repositioning of one of the dots. A message on the screen instructed the subject to press the $S$ key if he or she thought the second display was the same as the first, or the D key if different. The subject was again permitted to correct the response prior to pressing the return key. Once this was done, the display disappeared, to be followed by the instruction to initiate the next trial.

\section{Procedure}

All subjects received all three conditions, in a counterbalanced order. There were thus three groups, each consisting of 5 subjects, 
with the 2 men in different groups; one group received the conditions in the order control/digits/dots, another in the order digits/dots/control, and the third in the order dots/control/digits.

Under each condition there were 10 practice trials, followed by 48 experimental trials in which the 48 possible letter stimuli were presented in random order. Subjects in the digits and dots conditions were told to give priority to these tasks rather than to the letter task, but still to try to respond quickly and accurately to the letters. They were told, however, that only the responses to the letters were being timed. In responding to the letters, they were told to use the forefingers of each hand, and to rest these fingers lightly on the $\mathrm{B}$ and $\mathrm{N}$ keys at the beginning of each trial.

Whenever the subject made an error on the mental-rotation task, the stimulus was repeated at the end of the sequence. Four subjects ( 1 man and 3 women) were replaced because they made 10 or more errors on at least one sequence of 48 experimental trials. Of the remaining subjects, the maximum number of errors on a sequence was 8 .

\section{RESULTS}

\section{Mental-Rotation Task}

For the mental-rotation task, the mean numbers of errors were $0.60,1.67$, and 1.47 , each out of 48 , under the control, digits, and dots conditions, respectively. An analysis of variance (ANOVA), in which the factors were order and condition, showed that these means did not differ significantly $[F(2,12)=1.99, M S e=2.59]$ and that no other effects were significant. The errors were too few to permit further statistical analyses.

Reaction times (RTs) were recorded in milliseconds for each correct response. These were then subjected to an ANOVA in which the between-subjects factor was order and the within-subjects factors were condition, letter, angular orientation, and version (normal vs. backward).

The significant main effects were those of condition $[F(2,24)=14.38, M S e=1,383,060, p<.01]$, letter $[F(3,36)=5.21, M S \mathrm{e}=868,034, p<.01]$, angular orientation $[F(5,60)=30.77, M S e=786,750, p<.01]$, and version $[F(1,12)=21.91, M S e=1,119,095$, $p<.01]$. The only interaction to reach significance was that between groups and conditions $[F(4,24)=14.68, M S \mathrm{e}$ $=1,383,060, p<.01]$; this interaction was attributable to a decrease in RT over successive blocks of trials.

The mean RTs to the four letters were $1,104 \mathrm{msec}$ to the F, $1,252 \mathrm{msec}$ to the $\mathrm{L}, 1,225 \mathrm{msec}$ to the $\mathrm{P}$, and $1,064 \mathrm{msec}$ to the $R$. The version effect was due to shorter RTs for normal $(1,055 \mathrm{msec})$ than for backward $(1,268 \mathrm{msec})$ letter versions.

Mean RT was longest for the digits condition $(1,327$ msec), next longest for the patterns condition $(1,225$ $\mathrm{msec}$ ), and shortest for the control condition (995 msec). Scheffé tests revealed each pairwise comparison to be significant $(p<.01)$, suggesting that the mental-rotation task did compete for attentional resources with the two short-term retention tasks. Retention of the digits made greater competing demands than did retention of the dot patterns.

The interaction between conditions and orientations was not significant, however $[F(10,120)=1.21, M S \mathrm{e}=$ $351,396]$. At this level, then, the analysis fails to support the hypothesis that the rate of mental rotation would be influenced by the competing tasks. The functions relating RT to angular orientation are plotted for each condition in Figure 1.

In order to assess more precisely the effect of condition on the rate of mental rotation, the effect of angular orientation was expressed as a contrast representing angular departure from the normal upright, and the interaction of this contrast with conditions was also insignificant $[F(2,120)=1.12, M S \mathrm{e}=351,396]$. According to this more exacting test, therefore, the data still fail to show any reliable effect of the competing conditions upon rate of rotation. Nevertheless, estimates of rate based on the least squares fit of the contrast to the mean RTs did reveal slight differences; the estimated rate was 286 degrees per second (dps) under the control condition, 249 dps under the digits condition, and $232.5 \mathrm{dps}$ under the patterns condition.

Inspection of Figure 1 suggests that these different estimates may have been due in part to differential flattening of the functions for orientations close to the normal upright; note that under the control condition, the mean RT is actually shorter at the $300^{\circ}$ orientation than at the $0^{\circ}$ orientation. This flattening effect is commonly observed in studies of mental rotation, and has been interpreted to mean that subjects do not always mentally rotate the characters the full angular distance to the upright (Cooper \& Shepard, 1973; Hock \& Tromley, 1978). It is less marked in the patterns condition than in the control condition, and less marked still in the digits condition. Since this effect tends to increase the estimated rate of mental rotation, it may partially explain the small differences in estimated rate between conditions.

In order to pursue this point, the simple main effect of orientation was assessed separately for each condition; the error term for testing hypotheses about these simple main effects and degrees of freedom were calculated according to the formulas given in Winer (1971). Under the con-

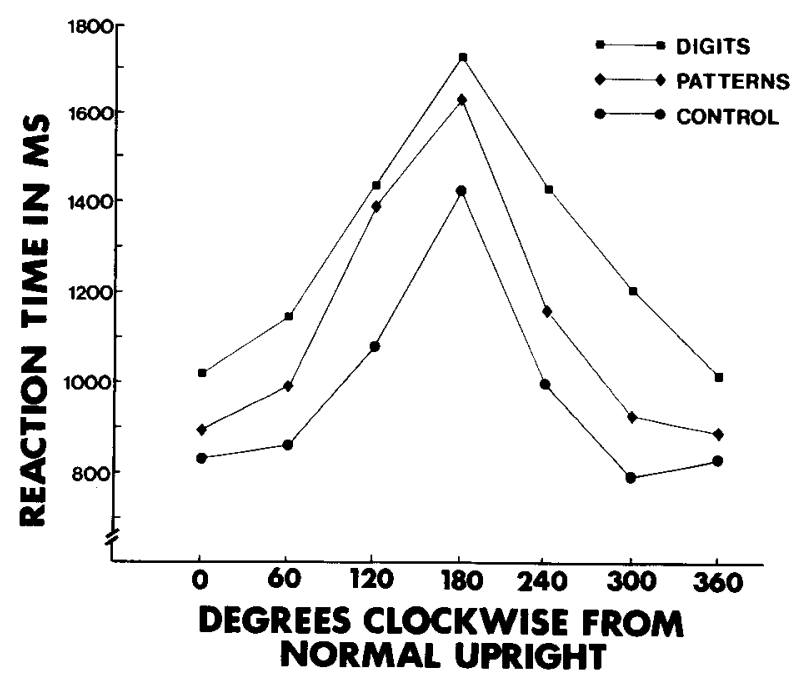

Figure 1. Mean reaction time at each angular orientation under each condition. 
trol condition, the contrast representing mental rotation accounted for $82.99 \%$ of the variance between orientations, and the deviation was in fact significant $[F(4,154)$ $=2.969, M S e=496,514, p<.05]$. This deviation can be attributed primarily to the flattening effect close to the $0^{\circ}$ orientation. Under the digits condition, on the other hand, the contrast accounted for $97.20 \%$ of the variance, and the deviation was not significant $[F(4,154)=0.55$, $M S \mathrm{e}=496,514]$. Under the patterns condition, the contrast accounted for $86.12 \%$ of the variance; the deviation was significant $[F(4,154)=3.56, M S e=496,514$, $p<.05$ ], but this is due in part to the marked asymmetry in the function, evident in Figure 1, as well as to the flattening effect.

Because mental rotation seems to have been at least curtailed within angular distances of $60^{\circ}$ from the upright, mental-rotation rates were reestimated by excluding the $0^{\circ}$ point and fitting a contrast representing mental rotation to the remaining five points. This procedure effectively estimates the rate of mental rotation from the given orientation to an orientation $60^{\circ}$ from the upright. For the control condition, the contrast accounted for $94.38 \%$ of the variance and resulted in an estimated rate of $208 \mathrm{dps}$. For the digits condition, the contrast accounted for $99.03 \%$ of the variance, and yielded an estimated rate of $218 \mathrm{dps}$, slightly faster than that under the control condition. For the patterns condition, the variance accounted for was $91.50 \%$ and the estimated rate was $181 \mathrm{dps}$.

In summary, the results suggest that the digits condition, which had the largest effect on overall RT in the mental-rotation task, nevertheless had no influence on the rate of mental rotation. However, the subjects seem to have been more likely to mentally rotate the letters the full angular distance to the upright under the digits than under the control condition; perhaps the option of stopping mental rotation at some angular distance from the upright is one that draws on attentional resources (cf. Logan \& Cowan, 1984). The estimated rate of rotation under the patterns condition remained slightly below that of the control and digits condition, even after correction for the flattening of the function. Although there were no statistical grounds for supposing this effect to have been reliable, it may reflect competition for resources within the spatial domain.

\section{Digits Task}

The numbers of digits reported in their correct positions under the digits condition were analyzed as a function of the parameters of the mental-rotation task, namely, letter, angular orientation, and version. There were no effects even approaching significance; in particular, the effect of angular orientation was negligible $[F(5,60)=$ $0.46, M S e=1.7424]$. This null result offers further evidence that the actual mental-rotation component of the mental-rotation task did not compete with the digits task for attentional resources. Performance on the digits task was sufficiently close to the expected memory span, yet sufficiently removed from the ceiling, to suggest that it
Table 1

Mean Number of Digits Correctly Reported and Mean

Proportion of Dot Configurations Correctly Recognized

\begin{tabular}{lcccccc}
\hline & \multicolumn{6}{c}{ Angular Orientation } \\
\cline { 2 - 7 } & $0^{\circ}$ & $60^{\circ}$ & $120^{\circ}$ & $180^{\circ}$ & $240^{\circ}$ & $300^{\circ}$ \\
\hline $\begin{array}{l}\text { Number } \\
\text { Digits Correct }\end{array}$ & 6.59 & 6.63 & 6.52 & 6.73 & 6.62 & 6.50 \\
$\begin{array}{l}\text { Proportion } \\
\text { Dots Correct }\end{array}$ & .808 & .817 & .817 & .783 & .825 & .783 \\
\hline
\end{tabular}

did tax central resources to the limit. Table 1 shows the mean number correct at each orientation.

\section{Dots Task}

Table 1 also shows the mean proportions of correct responses in the dots task as a function of the angular orientation of the letters in the mental-rotation task. This effect also proved insignificant $[F(5,60)=0.34, M S e=$ 0.1160 ]. Performance was well above chance $(0.5)$, yet clearly below ceiling, suggesting that this task also taxed central resources.

\section{DISCUSSION}

The results are similar to those reported by Logan (1978) on the effects of short-term retention on memory scanning. They show an overall effect of memory load on the time taken to perform the mental-rotation task, but little or no effect on the mental-rotation component itself. Conversely, performance on the memory tasks was not influenced by the degree of inferred mental rotation. These findings are in line with Logan's view that attentional control is involved in the setting up or harnessing of processing structures involved in a task, but not in the actual execution of the component processes. The fact that the retention tasks did increase overall $\mathrm{RT}$ to the mentalrotation task, even though they did not increase the rate of mental rotation, suggests that they did compete for attentional resources; this competition probably resulted from rehearsal of the material in short-term memory rather than from retention per se.

Although the results can be taken to mean that the mental-rotation component does not require attentional resources, it might also be argued that the subjects switched attention from the retention of the digits or dots to the mental-rotation task. This could have produced a delay in overall RT to perform the mental rotation, but allowed the subjects to allocate full attentional resources to the actual mental-rotation component. However, if this were so, one might have expected retention to decline with the angle of mental rotation, but in neither retention task was there any evidence that this was the case. Even so, it remains possible that the varying delays introduced by mental rotation may have been too small to materially affect short-term retention. There is evidence, however, that the influence of delay is maximal immediately after presentation of the material, and increases as the number of items in memory approaches the memory span (Mel- 
ton, 1963). In these respects, the conditions in the present experiment should have been nearly optimal for detecting any effects of delay due to the switching of attention, yet no such effects were observed.

The more likely explanation of our results, then, is that the actual mental rotation of the letters was accomplished in automatic rather than in controlled mode. Yet, mental rotation also has some of the characteristics of a controlled process, in that subjects seem aware of it and are able to initiate it, and even control its rate, according to instructions (Cooper \& Shepard, 1973). The results, therefore, add to the growing evidence that the distinction between controlled and automatic processing may not represent a true dichotomy. Logan and Cowan (1984) have also argued that many automatic processes are controlled, in the sense that they are serial, organized by thought, and directed toward a goal. The critical question is whether this control involves executive or subordinate systems. Executive control is attention-demanding, and is thus subject to interference from other attentiondemanding activities. However, many serial processes are serially organized and goal-directed, yet are controlled by subordinate systems that do not require attention.

Everyday activities, such as driving a car or walking down a busy street, may involve intricate planning and control, yet they seldom call upon the executive system. The executive can take over, however, if unexpected contingencies arise, or even as a matter of whim. Mental rotation may well fall into the category of serial processes that can be relegated to subordinate control, especially in the context of experiments in which subjects are repeatedly required to make simple decisions requiring mental rotation. Yet the executive can assume control if the situation demands it, as when subjects are explicitly instructed to carry out mental rotations according to specifications (see Cooper \& Shepard, 1973).

There is one respect in which the competing tasks did seem to influence mental rotation. Under the competing influence of the digits task, the subjects seemed more likely to rotate the letters the full angular distance to the upright, whereas in the control condition, mental rotation apparently stopped in the vicinity of $60^{\circ}$ from the normal upright. A possible explanation for this is that attentional control is required in order to stop rotation at an orientation other than the normal upright (see Logan \& Cowan, 1984). Alternatively, the explanation may lie at the decision stage rather than the mental-rotation stage. In deciding whether a letter is normal or backward, subjects may be able to tolerate a greater degree of tilt if more attentional resources are available. If there is competition for resources, then subjects may be obliged to mentally rotate the letters closer to the normal upright.

The results do not bear strongly on the question of modularity (Fodor, 1983). Although the estimated rates of mental rotation did not differ significantly between conditions, it is perhaps worth noting that the estimated rate was slowest under the patterns condition, even though overall RT was longest under the digits condition. This might be taken as weak evidence that there was some "module-specific" interference between retention of the patterns and the act of mental rotation itself. The asymmetry in the orientation function under the patterns condition also suggests that mental rotation was slower when the letters were oriented at $60^{\circ}$ and $120^{\circ}$ than at $300^{\circ}$ and $240^{\circ}$, respectively, implying slower rotation counterclockwise than clockwise. There seems no obvious reason, however, why spatial interference should have created such an asymmetry.

\section{REFERENCES}

COOPER, L. A., \& SHEPARD, R. N. (1973). Chronometric analyses of the rotation of mental images. In W. G. Chase (Ed.), Visual information processing (pp. 75-176). New York: Academic Press.

Corballis, M. C., KaTZ, J., \& Schwartz, M. (1980). Retrieval from memory sets that exceed the memory span. Canadian Journal of Psychology, 34, 40-48.

Corballis, M. C., Kirby, J., \& Miller, A. (1972). Access to elements of a memorized list. Journal of Experimental Psychology, 94, 185-190.

FODOR, J. A. (1983). The modularity of mind: An essay on faculty psychology. Cambridge, MA: The MIT Press/Bradford Books.

Hock, H. S., \& Tromley, C. L. (1978). Mental rotation and perceptual uprightness. Perception \& Psychophysics, 24, 529-533.

KlapP, S. T., Marshburn, E. A., \& Lester, P. T. (1983). Short-term memory does not involve the "working memory" of information processing: The demise of a common assumption. Joumal of Experimental Psychology: General, 112, 240-264.

KRISTOFFERSON, M. W. (1977). The effects of practice with one positive set in a memory scanning task can be completely transferred to a different positive set. Memory \& Cognition, 5, 177-186.

LOGAN, G. D. (1978). Attention in character-classification tasks: Evidence for the automaticity of component stages. Joumal of Experimental Psychology: General, 107, 32-63.

LOGAN, G. D., \& COWAN, W. B. (1984). On the ability to inhibit thought and action: A theory of an act of control. Psychological Review, 91, 295-327.

MeLton, A. W. (1963). Implications of short-term memory for a general theory of memory. Journal of Verbal Learning \& Verbal Behavior, 2, 1-21.

RatCLif, R. (1978). A theory of memory retrieval. Psychological Review, 85, 59-108.

RYAN, C. (1983). Reassessing the automaticity-control distinction: Item recognition as a paradigm case. Psychological Review, 90, 171-178.

SCHNEIDER, W., \& SHIFFrIN, R. M. (1977). Controlled and automatic human information processing: I. Detection, search, and attention. Psychological Review, 84, 1-66.

SHiffrin, R. M., \& SCHNeIDER, W. (1977). Controlled and automatic human information processing: II. Perceptual learning, automatic attending, and a general theory. Psychological Review, 84, 127-190.

Shulman, H. G., \& Greenberg, S. N. (1971). Perceptual deficit due to division of attention between memory and perception. Journal of Experimental Psychology, 88, 171-176.

Shulman, H. G., Greenberg, S. N., \& Martin, J. (1971). Intertask delay as a parameter of perceptual deficit in divided attention. Journal of Experimental Psychology, 88, 439-440.

STERNBERG, S. (1966). High-speed scanning in human memory. Science, $153,652-654$.

STERNBERG, S. (1969). The discovery of processing stages: Extensions of Donders' method. In W. G. Koster (Ed.), Attention and performance II (pp. 276-315). Amsterdam: North-Holland.

WINER, B. J. (1971). Statistical principles in experimental design. New York: McGraw-Hill.

(Manuscript received July 15, 1985; revision accepted for publication October 2, 1985.) 